\title{
Analysis of defect related optical transitions in biased $\mathrm{AlGaN} / \mathrm{GaN}$ heterostructures
}

\author{
A. Bengi ${ }^{\text {a,* }}$, S.B. Lisesivdin ${ }^{\text {b }}$, M. Kasap ${ }^{\text {a }}$, T. Mammadov ${ }^{\text {a,c }}$, S. Ozcelik ${ }^{\text {a }}$, E. Ozbay ${ }^{\text {b,d,e }}$ \\ a Physics Department, Faculty of Arts and Sciences, Gazi University, 06500 Ankara, Turkey \\ ${ }^{\mathrm{b}}$ Nanotechnology Research Center, Bilkent University, 06800 Ankara, Turkey \\ c Institute of Physics, National Academy of Science, Baku, Azerbaijan \\ d Department of Physics, Bilkent University, 06800 Ankara, Turkey \\ e Department of Electrical and Electronics Engineering, Bilkent University, 06800 Ankara, Turkey
}

\section{A R T I C L E I N F O}

\section{Available online 8 June 2010}

\section{Keywords:}

AlGaN/GaN

MOCVD

2DEG

Photoluminescence

Under bias

\begin{abstract}
A B S T R A C T
The optical transitions in AlGaN/GaN heterostructures that are grown by metalorganic chemical vapor deposition (MOCVD) have been investigated in detail by using Hall and room temperature (RT) photoluminescence (PL) measurements. The Hall measurements show that there is two-dimensional electron gas (2DEG) conduction at the AlGaN/GaN heterointerface. PL measurements show that in addition to the characteristic near-band edge (BE) transition, there are blue (BL) and yellow luminescence (YL) bands, freeexciton transition (FE), and a neighboring emission band (NEB). To analyze these transitions in detail, the PL measurements were taken under bias where the applied electric field changed from 0 to $50 \mathrm{~V} / \mathrm{cm}$. Due to the applied electric field, band bending occurs and NEB separates into two different peaks as an ultraviolet luminescence (UVL) and $\mathrm{Y}_{4}$ band. Among these bands, only the yellow band is unaffected with the applied electric field. The luminescence intensity change of these bands with an electric field is investigated in detail. As a result, the most probable candidate of the intensity decrease with an increasing electric field is the reduction in the radiative lifetime.
\end{abstract}

(c) 2010 Elsevier Ltd. All rights reserved.

\section{Introduction}

Group-III-nitride semiconductors and their alloys were studied extensively and used for optoelectronic [1,2] and electrical applications [3,4]. Due to the respective advantages such as high breakdown voltage, electron peak velocity, and high electron density, AlGaN/GaN heterostructures are advantageous for high-frequency and high-power applications at higher temperatures when compared with Si- and GaAs-based devices [5,6].

Because of the insufficient high quality lattice-matched substrates, AlGaN/GaN heterostructures are mostly grown on sapphire substrate, which causes a high number of

\footnotetext{
* Corresponding author. Tel.: +903122021279.

E-mail address: aylinbengi@gmail.com (A. Bengi).
}

dislocations. Dislocation related structural defects and other point defects (native defect, impurities, and complexes) notably affect the optical and electrical properties of the material [7]. Degradation of the performance and reliability of the group-III nitride semiconductor devices, originating from point defects, has been reported by many authors $[8,9]$. An in-depth understanding of point defects will lead to further improvements in device performance and reliability. Photoluminescence (PL) is one of the advanced defect detection and identification methods for semiconductors and has been extensively used for wide band-gap semiconductors when the electrical characterization is limited because of large activation energies beyond the thermal activation limits [10]. Recently, the luminescence properties of defects in GaN-systems have been reviewed extensively by Reschikov and Morkoç [10]. To investigate defect 
related electronic and optical properties in detail, one of the promising optical characterization methods is taking the PL measurements under bias [11,12].

In the present work, we investigated the electrical and optical properties of AlGaN/GaN heterostructures by using Hall and room temperature PL measurements. Defect related optical transitions were investigated by using RT PL measurements under bias where the applied electric field changed from 0 to $50 \mathrm{~V} / \mathrm{cm}$.

\section{Experimental details}

The investigated samples were grown on $c$-plane (0001) sapphire $\left(\mathrm{Al}_{2} \mathrm{O}_{3}\right)$ substrate in a low pressure MOCVD reactor. Before the epilayer growth, the sapphire substrate was cleaned in $\mathrm{H}_{2}$ ambient at $1100{ }^{\circ} \mathrm{C}$, and then a $25 \mathrm{~nm}$-thick low temperature (LT) GaN nucleation layer was grown at $500{ }^{\circ} \mathrm{C}$. The reactor pressure was set to 50 mbar during the substrate cleaning and nucleation growth. After the deposition of the LT-GaN nucleation layer, the wafer was heated to a high-temperature (HT) for annealing. The two-step growth process parameters of the ramp time and annealing temperature were $5 \mathrm{~min}$ and $1050{ }^{\circ} \mathrm{C}$, respectively. Approximately a $2.5 \mu \mathrm{m}$ thick GaN layer was deposited on the annealed nucleation layers by using constant growth conditions. Finally, a $25 \mathrm{~nm}$ thick $\mathrm{Al}_{0.25} \mathrm{Ga}_{0.75} \mathrm{~N}$ with a $3 \mathrm{~nm}$ GaN cap layer was grown. The layer structure of the samples is shown in Fig. 1 inset.

Hall Effect measurements were performed by using the van der Pauw (vdP) method. For the electrical measurements, the square shaped $\left(5 \times 5 \mathrm{~mm}^{2}\right)$ samples were prepared with $\mathrm{Ti} / \mathrm{Al} / \mathrm{Ni} / \mathrm{Au}$ evaporated dot contacts in the corners. The ohmic behavior of the contacts was confirmed by the current voltage $(I-V)$ characteristics. The measurements were performed at a $0.5 \mathrm{~T}$ magnetic field (with $0.1 \%$ uniformity) in a temperature range $20-350 \mathrm{~K}$ by using a Lakeshore Hall Effect Measurement System (HMS). At each temperature step, the Hall coefficient (with a maximum 5\% error) and resistivity (with a maximum $0.2 \%$ error in the studied range) were measured

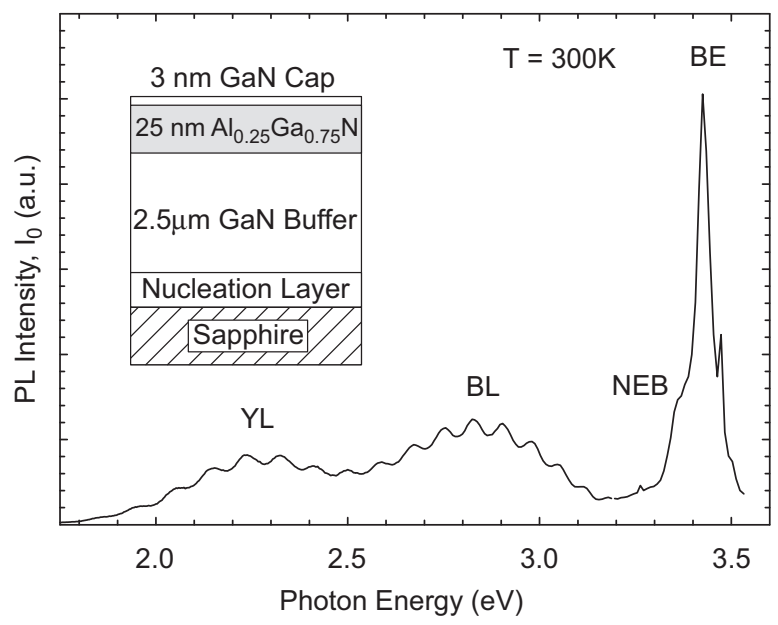

Fig. 1. PL spectrum of the $\mathrm{AlGaN} / \mathrm{GaN}$ heterostructure at $300 \mathrm{~K}$. Inset: layer structure of the investigated samples. and then the Hall mobility and Hall sheet carrier density values were calculated. The PL measurements were carried out at room temperature under a variable electric field $(0-50 \mathrm{~V} / \mathrm{cm})$, which was applied to the opposite corners of the vdP sample. PL measurements were taken from the center of the sample where the maximum electric field intensity existed. For the PL measurements, the Horiba Jobin Yvon Fluorolog 3 spectrofluorometer with $325 \mathrm{~nm}$ ultraviolet light emitted from a $50 \mathrm{~mW}$ He-Cd laser excitation source was used.

\section{Results and discussion}

The room temperature PL spectrum of the AlGaN/GaN heterostructure is shown in Fig. 1. The spectrum shows three main transitions, namely near-band edge (BE) transition, one broad blue luminescence (BL) band, and another broad yellow luminescence (YL) band centered at $3.42,2.83$, and $2.27 \mathrm{eV}$, respectively. There are fringes on the YL and $\mathrm{BL}$ bands, due to the multiple reflection of the laser light between the epilayers. In addition to these main transitions, neighboring emission band (NEB) that appears as a shoulder on the BE peak and free-exciton transition (FE) at $3.47 \mathrm{eV}$ can be clearly seen in the figure.

The transition energies depend on the point defects such as the impurities that generally originate from unintentional doping and native defects. Generally, $\mathrm{Si}$ and $\mathrm{C}$ on the Ga sites $\left(\mathrm{Si}_{\mathrm{Ga}}\right.$ and $\left.\mathrm{C}_{\mathrm{Ga}}\right)$ and $\mathrm{O}$ on the $\mathrm{N}$ sites $\left(\mathrm{O}_{\mathrm{N}}\right)$ are considered as shallow donors and $\mathrm{Zn}, \mathrm{Be}$, and $\mathrm{Mg}$ on the $\mathrm{Ga}$ sites $\left(\mathrm{Zn}_{\mathrm{Ga}}, \mathrm{Be}_{\mathrm{Ga}}, \mathrm{Mg}_{\mathrm{Ga}}\right)$ and $\mathrm{Si}$ and $\mathrm{C}$ on the $\mathrm{N}$ sites $\left(\mathrm{Si}_{\mathrm{N}}, \mathrm{C}_{\mathrm{N}}\right)$ are considered as shallow acceptors in GaN [10]. Due to the low formation energies of $\mathrm{Si}_{\mathrm{Ga}}$ and $\mathrm{O}_{\mathrm{N}}$, these are the most probable impurity types in GaN. Native defects such as vacancies are related to non-stoichiometric growth or intentional doping, and significantly affect the optical properties of the material. In the $n$-type $\mathrm{GaN}$, a gallium vacancy $\left(V_{\mathrm{Ga}}\right)$ is the dominant native defect, while the nitrogen vacancy $\left(V_{N}\right)$ is the same as that in p-type GaN. It is well known that undoped GaN usually has $n$-type conductivity and gallium vacancy acts as an acceptor-like native defect which is responsible for this conductivity [13]

The BE transition is related to shallow donor bound exciton (DBE) and generally shows a sharp peak close to the energy gap of the semiconductor [14]. The dominant mechanism for BE luminescence is the formation of shallow donor due to $V_{N}[15]$ and $O_{N}[16]$.

Another emission band, called $\mathrm{BL}$, which is centered at $2.83 \mathrm{eV}$ in our case, sometimes appears in unintentionally doped GaN films at room temperature [17-19] and is related to the crystalline quality. The formation of a BL band is due to the recombination of the free electron in a conduction band (around room temperature) to relatively deep acceptor (e-A luminescence) levels. The observation of BL is typical for MOCVD grown GaN layers [18] and is related to some impurities coming from an MO source ( $\mathrm{Mg}, \mathrm{Zn}$ ). The $\mathrm{BL}$ is dominated by the involvement of oxygen, carbon, and hydrogenated gallium vacancies in $n$-type GaN [20] and is associated with some metastable defects [21]. In our previous work, the presence of high concentrations of carbon and oxygen impurities in similar structures were shown by using the SIMS technique [22].

Besides for other emission bands, the most important one is the YL band that is centered at $2.27 \mathrm{eV}$, which occurs in nearly all GaN samples. Its position and shape are somewhat sample dependent [10] and strongly limit the optical applications of these materials [23]. The formation of this band is related to the transition of electrons from the conduction band to the deep acceptor states. These states are the evidence of native defects and residual impurities (compensating centers) [23-25] due to system contamination and background carrier concentration related to high-temperature GaN growth conditions. YL is not related to the specific impurity, wherein the most probable candidate is the deep acceptor $\left(C_{N}\right)$ assisted gallium vacancy $\left(V_{\mathrm{Ga}} C_{\mathrm{N}}\right)$ $[10,26]$, in which it is believed that the presence of higher carbon impurity concentration in highly resistive films is an evidence of the reduced growth temperatures [27]. Even though the main mechanism behind the YL formation is unknown, there is much evidence that the YL originates from the GaN/sapphire interface. 
To understand the main conduction mechanism, temperature dependent Hall mobility and sheet carrier density measurements were performed. Fig. 2 shows the temperature dependence of Hall mobility and the sheet carrier density. For the investigated samples, Hall mobility and the measured sheet electron density are nearly independent of temperatures below $100 \mathrm{~K}$. This behavior is typical of 2DEG structures. At higher temperatures, optical phonon scattering is dominant and, therefore, Hall mobility dramatically decreases with increasing temperature. The sheet carrier density still tends to be a constant which is a further confirmation of the 2DEG, even at high temperatures. Room temperature Hall mobility and the measured sheet carrier density are $719 \mathrm{~cm}^{2} / \mathrm{V}$.s and $1.50 \times 10^{13} \mathrm{~cm}^{-2}$, respectively. The high values of the sheet carrier density are caused by spontaneous and piezoelectric polarization fields [28]. At $20 \mathrm{~K}$, the Hall mobility is as high as $3780 \mathrm{~cm}^{2} / \mathrm{V} . \mathrm{s}$ These results show that a nominal AlGaN/GaN heterointerface with dominant 2DEG conduction were grown on sapphire substrates.

In addition to the conventional PL measurements, the same measurements were carried out under bias at room temperature. The bias voltage was applied to opposite corners of the vdP samples where the applied electric field changed from 0 to $50 \mathrm{~V} / \mathrm{cm}$. PL spectra under bias are shown in Fig. 3. The PL intensity was directly affected by the point defects and surface states by varying the radiative recombination probability or indirectly via varying the band bending. As can be seen in Fig. 3, among all four transitions, only the YL was unaffected by the applied electric field. The intensity of the BL decreases more significantly than BE with the applied electric field. The NEB becomes clear and slightly decreases with increasing applied electric field.

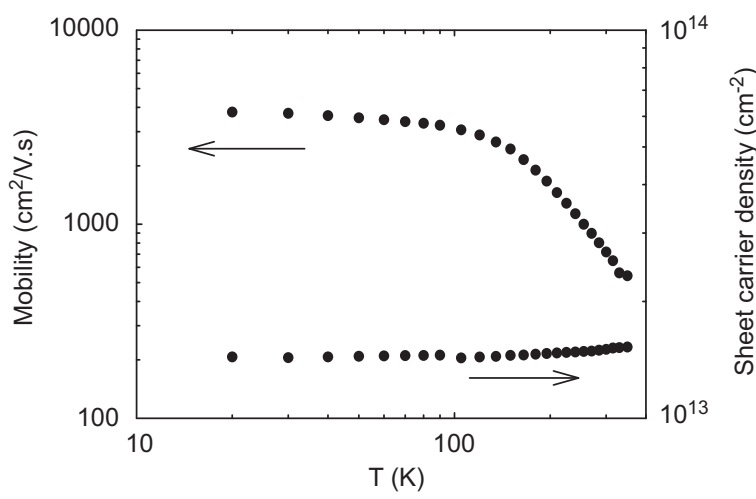

Fig. 2. Temperature dependent Hall mobility and sheet carrier density.

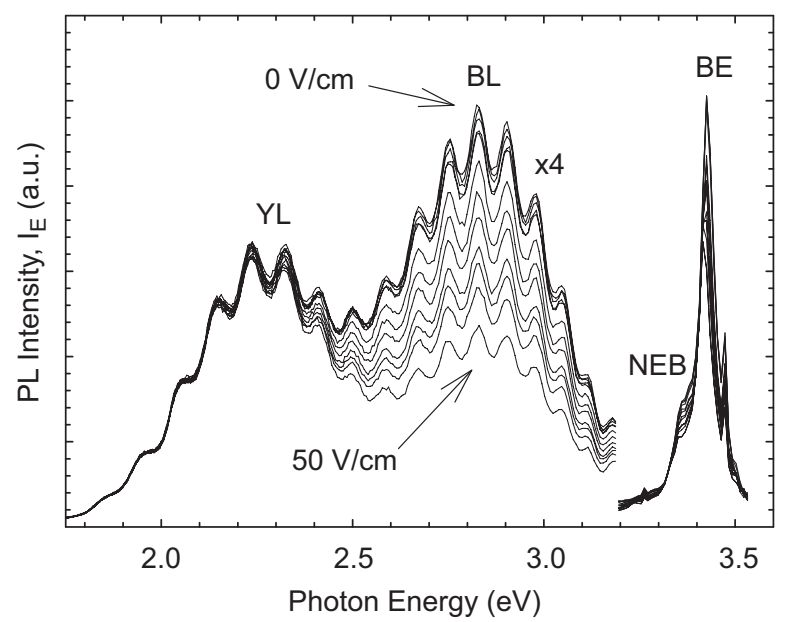

Fig. 3. Electric field dependent PL spectrum of the AlGaN/GaN heterostructure at $300 \mathrm{~K}$.

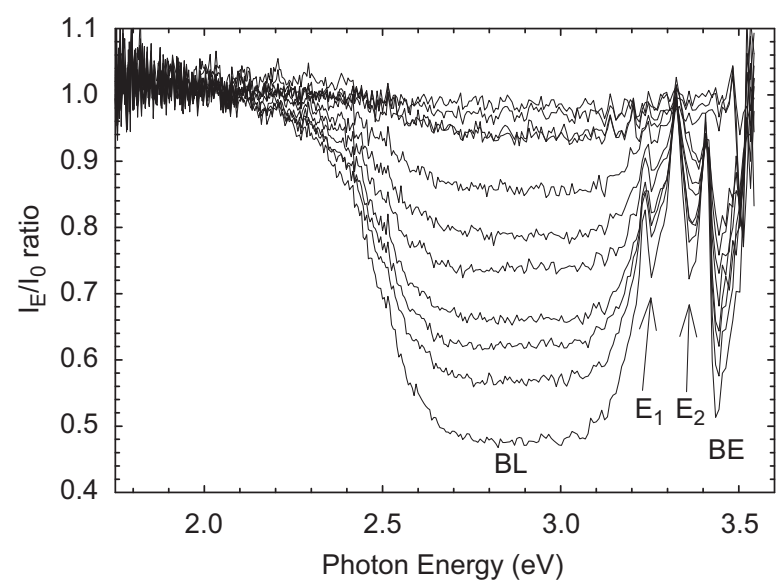

Fig. 4. Normalized $\left(I_{\mathrm{E}} / I_{\mathrm{O}}\right)$ PL spectra (PL spectra are normalized by an unbiased PL spectrum intensity $\left(I_{\mathrm{o}}\right)$ ).

To see the effects of the applied electric field on the transitions, the spectra are normalized by the zero field PL spectrum intensity $\left(I_{0}\right)$ as shown in Fig. 4. When the normalized intensities of the transitions are compared, $\mathrm{BE}$ and $\mathrm{BL}$ are the lowest in the entire spectrum. Due to the normalized intensity of the BE, it is slightly higher than BL, by which it can be suggested as the binding energy that is necessary for the dissociation of the electron-hole pairs of the BL emissions that may be slightly lower than that of the $\mathrm{BE}$ emissions. This BE behavior is explained as the probability of bound and free-exciton transitions being reduced, where the BL behavior can be explained as the carriers in the deep acceptor levels being transited to the conduction band by PL excitation. Moreover, the free photogenerated electrons and holes are accelerated due to the applied electric field, by which they interact with the bound and free excitons and carriers in the deep acceptors. Due to these interactions, the radiative recombination probability decreases, and these electrons in turn contribute to 2DEG conductivity at the AlGaN/GaN interface. As a result, the PL intensity decreases with the increasing electric field [12]. The YL forms at the GaN/sapphire interface, in which the carriers at this interface are not related to 2DEG formation at the AlGaN/GaN interface. Thus, there is no change in the recombination probability and, therefore, the intensity of the YL is independent of the applied electric field.

In Fig. 4, NEB separates into two different peaks, namely $E_{1}$ peak centered at $3.25 \mathrm{eV}$ and $E_{2}$ peak centered at $3.36 \mathrm{eV}$. The $E_{1}$ peak is attributed to the ultraviolet luminescence (UVL) band and at room temperature it is due to the transition from the shallow donor to the shallow acceptor. That is, the free electrons that are thermally released from the shallow donor $\left(\mathrm{Si}_{\mathrm{Ga}}\right.$ and $\left.\mathrm{O}_{\mathrm{N}}\right)$ to the conduction band recombine with holes bound to the same acceptor. The main candidates for the shallow acceptor in an undoped GaN are not clear, but it is believed to be due to $\mathrm{Mg}$ contamination $\left(\mathrm{Mg}_{\mathrm{Ga}}\right)$ from the system [10]. The other peak $E_{2}$ is attributed to the $\mathrm{Y}_{4}$ band and, is due to surface-state of an exciton bound to a structural defect at the surface $[29,30]$, an exciton bound to some point defects that is trapped by the edge threading dislocations.

To investigate the effects of the applied electric field on the main bands, the bias dependent PL spectrum, as shown in Fig. 3, is fitted by using multiple Gaussian fit and the fringe free spectrum is thereby obtained. To understand the procedure, the fit process for a zero bias spectrum is shown as an example in Fig. 5. For the other bias steps, this procedure is repeated and the results are given in Fig. 5 inset. From Figs. 4 and 5 inset, the position of the peak maximum of each band remains nearly unchanged and the intensity of the BE and BL bands quench, while the YL band intensity remains unchanged. This clearly indicates that the BE and BL band were influenced by the applied electric fields.

The intensity quenching of the bands is due to four mechanisms. The most probable candidate is the reduction of the radiative lifetime: when the electric field is applied to the heterostructure, the band bending occurs and the photogenerated electron becomes delocalized. It breaks the exciton and contributes to the current. The second mechanism is the 


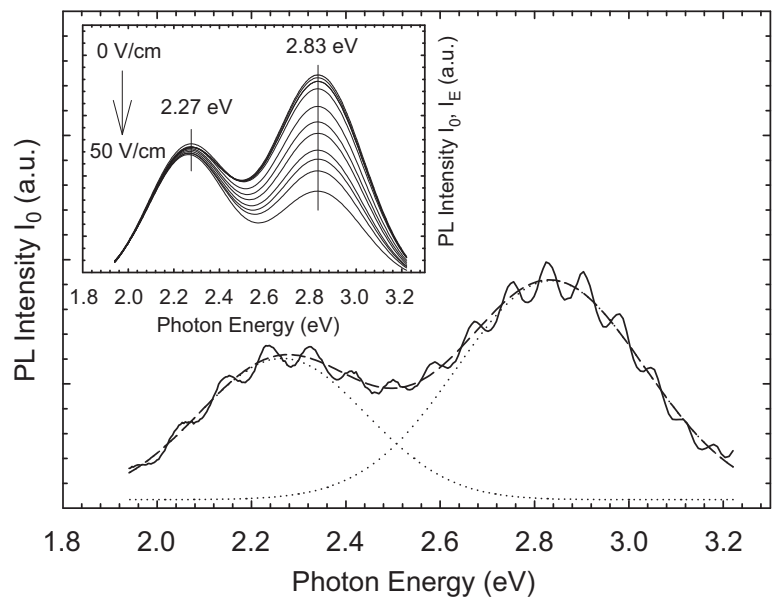

Fig. 5. Multiple Gaussian fit procedure for an unbiased PL spectrum. Inset: fringe free PL and PL under bias spectra. The peak positions are also shown.

spatial separation of the carriers [31]: if the applied electric field is strong enough to overcome the binding energy of the exciton, then the excitons are dissociated and become free carriers. Therefore, there is a decrease in the photoluminescence. The third mechanism is thermal dissociation: the temperature of the heterostructure increases with the applied electric field (Joule heating) and at the temperatures around room temperature, the exciton is thermally dissociated before recombination occurs. The last possible mechanism is the impact ionization by the accelerated electrons [32,33]. However, because the carriers must achieve an energy that is at least equal to or greater than the band-gap energy in order to undergo impact ionization, impact ionization does not occur at small electric field strengths as in our case [34].

\section{Conclusions}

In the present work, the optical transitions in AlGaN/ GaN heterostructures grown by MOCVD were investigated by using Hall and PL measurements. Hall measurements were carried out at a $0.5 \mathrm{~T}$ magnetic field in a temperature range $20-350 \mathrm{~K}$. The PL measurements were carried out at room temperature. From the PL analysis, three main transitions (BE, BL, and $\mathrm{YL}$ ) and a neighbor emission peak (NEB) as a shoulder on the near-band edge peak were obtained. To understand the main optical transition mechanisms in detail, PL measurements under bias were performed, while the applied electric field was changed from 0 to $50 \mathrm{~V} / \mathrm{cm}$. From the normalized PL spectra, it is seen that a neighbor emission peak splitted into UVL and $\mathrm{Y}_{4}$ peaks. Except for the YL band, all the other transitions are affected by an applied electric field, in which there is an intensity quenching. The intensity quenching of the bands is due to four mechanisms and can be explained in the order of their probability of occurrence as: (i) reduction in the radiative lifetime; (ii) thermal dissociation; (iii) spatial separation of carriers; and (iv) impact ionization. Among these mechanisms, the last one, impact ionization, was not observed in our experiments due to the necessity of much higher electric field that can provide the thermal energy in the order of band-gap energy, and the most probable candidate of the intensity quenching with an applied electric field is the reduction in the radiative lifetime. In addition, the defect related mechanisms of the $\mathrm{BE}, \mathrm{BL}, \mathrm{YL}$, $\mathrm{UVL}$, and $\mathrm{Y}_{4}$ transitions were also investigated in detail.

\section{Acknowledgements}

This work is supported by the State Planning Organization of Turkey under Grant no. 2001K120590, by the European Union under the projects EU-PHOME and EU-ECONAM, and TUBITAK under the Project nos. 106E198, 107A004, and 107A012. One of the authors (Ekmel Ozbay) acknowledges partial support from the Turkish Academy of Sciences.

\section{References}

[1] Razeghi M, Rogalski A. J Appl Phys 1996;79:1996.

[2] Akasaki I, Amanı H. J Appl Phys 1997;36:5293.

[3] Khan MA, Chen Q, Shun CJ, Shur M, Gelmont B. Appl Phys Lett 1995;67:1429.

[4] Redwing JM, Tischler MA, Flynn JS, Elhamri S, Ahoujja M, Newrock RS, et al. Appl Phys Lett 1996;69:963.

[5] Khan MA, Chen Q, Shur M, Mcdermott BT, Higgins JA. IEEE Electron Device Lett 1996;17:325.

[6] Wu YF, Keller BP, Keller S, Kapolnek D, Kozodoy P, DenBaars SP, et al. Appl Phys Lett 1996;69:1438.

[7] Ponce FA. In: Pearton SJ, editor. InGaN and related materials optoelectronic properties of semiconductors and superlattices. Amsterdam: Gordon and Breach Science Publishers; 1997.

[8] Parish G, Keller S, Denbaars SP, Mishra UK. In: Proceedings of the Ninth Biennial Workshop on Organometallic Vapor Phase Epitaxy, 2000.

[9] Wu YF, Keller BP, Keller S, Xu JJ, Thibeault BJ, Denbaars SP, et al. IEICE Trans Electron Devices 1999;E82-C:1895.

[10] Reshchikov MA, Morkoç H. J Appl Phys 2005;97:061301.

[11] Ulbrich RG. Solid State Electron 1978;21:51.

[12] Jeong TS, Han MS. J Korean Phys Soc 2005;46:968.

[13] Neugebauer J, Van de Wall CG. Phys Rev B 1994;50:8067.

[14] Dingle R, Ilegems M. Solid State Commun 1971;9:175.

[15] Pankove JI. Mater Res Soc Symp Proc 1990;162:515.

[16] Wiefert W, Fanzhelal R. Cryst Res Technol 1993;18:383.

[17] Sasaki T, Zembutsu S. J Appl Phys 1987;61:2533.

[18] Li S, Jiang F, Han G, Wang L, Xiong C, Peng X, et al. Mater Sci Eng B 2005;122:72.

[19] Li S, Jiang F, Han G, Wang L, Xiong C, Peng X, et al. J Lumin 2004;106:219.

[20] Kucheyev SO, Toth M. Appl Phys Lett 2001;79:2154.

[21] Dhar S, Ghosh S. Appl Phys Lett 2002;80:4519.

[22] Yu H, Ozturk MK, Ozcelik S, Ozbay E. J Cryst Growth 2006;293:273.

[23] Suski T, Perlin P. Appl Phys Lett 1995;67:2188.

[24] Mattila T, Nieminen RM. Phys Rev B 1997;55:9571.

[25] Schubert EF, Goepfert ID, Redwing JM. Appl Phys Lett 1997;71: 3224.

[26] Xing H, Keller S, Wu Y-F, Maccarty L, Smorchkova IP, Buttari D, et al. J Phys: Condens Matter 2001;13:7139.

[27] Pankove JI, Hutchby IA. J Appl Phys 1976;47:5387.

[28] Ambacher O, Foutz B, Smart J, Shealy JR, Weimann NG, Chu K, et al. J Appl Phys 2000;87:334.

[29] Mizutani T, Ohno Y, Akita M, Kishimoto S, Maezawa K. IEEE Trans Electron Devices 2003;50:2015.

[30] Reshchikov MA, Jasinski J, Liliental-Weber Z, Huang D, He L, Visconti P, et al. Physica B 2003;340:440

[31] Rothenberg E, Kazes M. Nano Lett 2005;5:1581.

[32] Rludau W, Wagner E. Phys Rev B 1976;13:5410.

[33] Duboz JY, Binet F. Mater Sci Eng B 1997;43:269.

[34] Kolnik J, Oguzman IH, Brennan KF. J Appl Phys 1997;81:726. 